\title{
Síndrome de Blesovsky Pseudotumor pulmonar infrecuente y subdiagnosticado
}

\author{
Blesovsky syndrome \\ Uncommon and underdiagnosed pulmonary pseudotumor
}

\author{
José Ignacio Valenzuela, Danny Novoa, Edgar Barrera, \\ Carmen gMez, Rodolfo Dennis• Bogotá, D.C. (Colombia)
}

\begin{abstract}
Resumen
Se presenta el caso de un hombre de 68 años inmunosuprimido, en periodo tardío postrasplante hepático por cirrosis alcohólica en quien se encuentra de manera incidental atelectasia redonda del pulmón en estudios imagenológicos. El paciente cursaba asintomático y como único hallazgo positivo al examen físico presentaba estertores finos basales. No fue posible encontrar factores etiológicos relacionados, por lo que se categorizó como un caso idiopático. (Acta Med Colomb 2013; 38: xx-xx).
\end{abstract}

Palabras clave: atelectasia pulmonar, inmunosupresión, pseudotumor pulmonar.

\section{Abstract}

We present the case of a 68 year old immunosuppressed man, in the late period after liver transplant for alcoholic cirrhosis and in whom incidentally imaging studies showed a lung round atelectasis. The patient was asymptomatic and the only positive finding on physical examination was fine basal rales. It was impossible to find related etiological factors, so the case was categorized as idiopathic. (Acta Med Colomb 2013; 38: xx-xx).

Keywords: pulmonary atelectasis, immunosuppression, pulmonary pseudotumor.
Dr. José Ignacio Valenzuela: Residente de Medicina Interna, Universidad del Rosario; Dres. Danny Novoa, Edgar Barrera, Carmen Gómez y Rodolfo Dennis: Departamento de Medicina Interna de la Fundación Cardioinfantil. Bogotá, D.C. (Colombia)

Correspondencia. Dr. José Ignacio Valenzuela.

E-mail: joseival@gmail.com

Recibido: 03/XI/2012 Aceptado: 18/IX/2013

\section{Introducción}

La atelectasia redonda del pulmón, conocida también como "pulmón plegado", "síndrome de Blesovsky", "atelectasia helicoidal", "pseudotumor atelectático, "pseudotumor pulmonar" y "pleuritis plegada con atelectasia", constituye un pseudotumor pulmonar raro comúnmente asociado con la exposición a asbesto y la enfermedad pleural relacionada $(1,2)$.

Fue descrita por primera vez por Loeschke en 1928 asociada a un derrame pleural (3), pero "popularizada" posteriormente por Hanke y Kretzschmaren 1980 quienes refinaron la descripción de sus características radiológicas y acuñaron el término "pseudotumor atelectásico del pulmón" (4),

Los hallazgos imagenológicos son característicos, y en ojos expertos, permiten establecer el diagnóstico (5). No obstante, se estima que por lo menos $50 \%$ no se diagnostican adecuadamente (6), tal vez porque realzan con la administración de medios de contraste, lo que dificulta su diferenciación con procesos neoplásicos malignos.

Se presenta el caso de un paciente inmunosuprimido con hallazgo incidental de atelectasia redonda, diagnosticada durante el periodo tardío postrasplante hepático, sin exposición previa a asbestos.

\section{Presentación del caso}

Paciente masculino de 68 años de edad, mestizo, natural y procedente de Chiquinquirá (Boyacá), agricultor y ganadero. Antecedente de tabaquismo con un índice calculado paquetes/año de cinco (suspendió hace 12 años), sin otros antecedentes exposicionales, sometido a trasplante hepático por cirrosis alcohólica hacía 13 meses, en manejo inmunosupresor con tacrolimus $3 \mathrm{mg}$ bid vía oral y micofenolato sódico $1.5 \mathrm{~g}$ bid vía oral, remitido de la consulta externa de hepatología por presentar estertores finos en base derecha, sin otros hallazgos positivos al examen físico. A la revisión por sistemas, se encontraba un paciente completamente asintomático.

Dado el estado de inmunosupresión del paciente, se iniciaron estudios para descartar un posible foco infeccioso (probable foco pulmonar), obteniéndose un hemograma sin leucocitosis, con trombocitopenia leve de 116000, VSG de 2 y radiografía de tórax anormal (Figura 1).

El paciente fue valorado por neumología, quienes solicitaron TAC de tórax de alta resolución (TACAR) para lograr una mejor caracterización de los hallazgos a la radiografía convencional, que reportó una masa de aproximadamente de $57 * 63 \mathrm{~mm}$ con arterias pulmonares en su interior y al- 

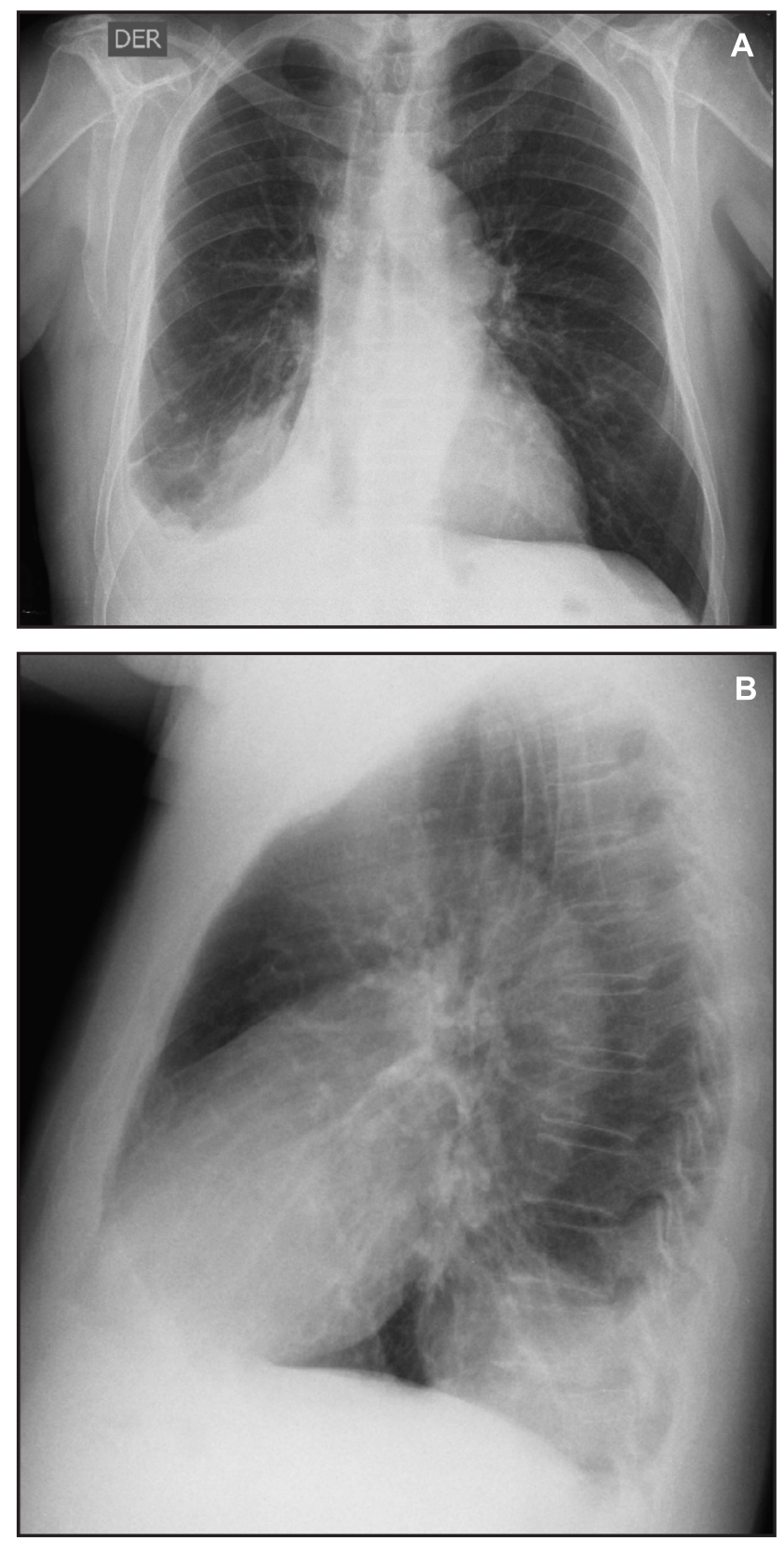

Figura 1. Radiografía de tórax posteroanterior y lateral que evidencia opacidad basal derecha en contacto con la pleura visceral, con presencia de broncograma aéreo, derrame pleural basal derecho asociado, tráquea desviada a la derecha con bronquio fuente derecho verticalizado y cisura mayor traccionada hacia abajo. Adicionalmente, nódulo pulmonar en lóbulo medio de $5.2 \mathrm{~mm}$ de diámetro.

rededor, en contacto con derrame pleural basal importante, compatible con el signo de la cola de cometa, estableciendo el diagnóstico de atelectasia redonda. Adicionalmente, un nódulo pulmonar en lóbulo medio (Figura 2).

Se extendió el estudio con una fibrobroncoscopia más lavado broncoalveolar (LBA) que reportó leucocitos 2530 por campo (neutrófilos 90\%, linfocitos 10\%), células epiteliales $>30$, con cultivos con crecimiento de Haemophilus spp $\left(10^{4} \mathrm{UFC} / \mathrm{ml}\right)$ multisensible y Moraxella group $\left(10^{4} \mathrm{UFC} / \mathrm{ml}\right)$ multisensible, que fue interpretado como
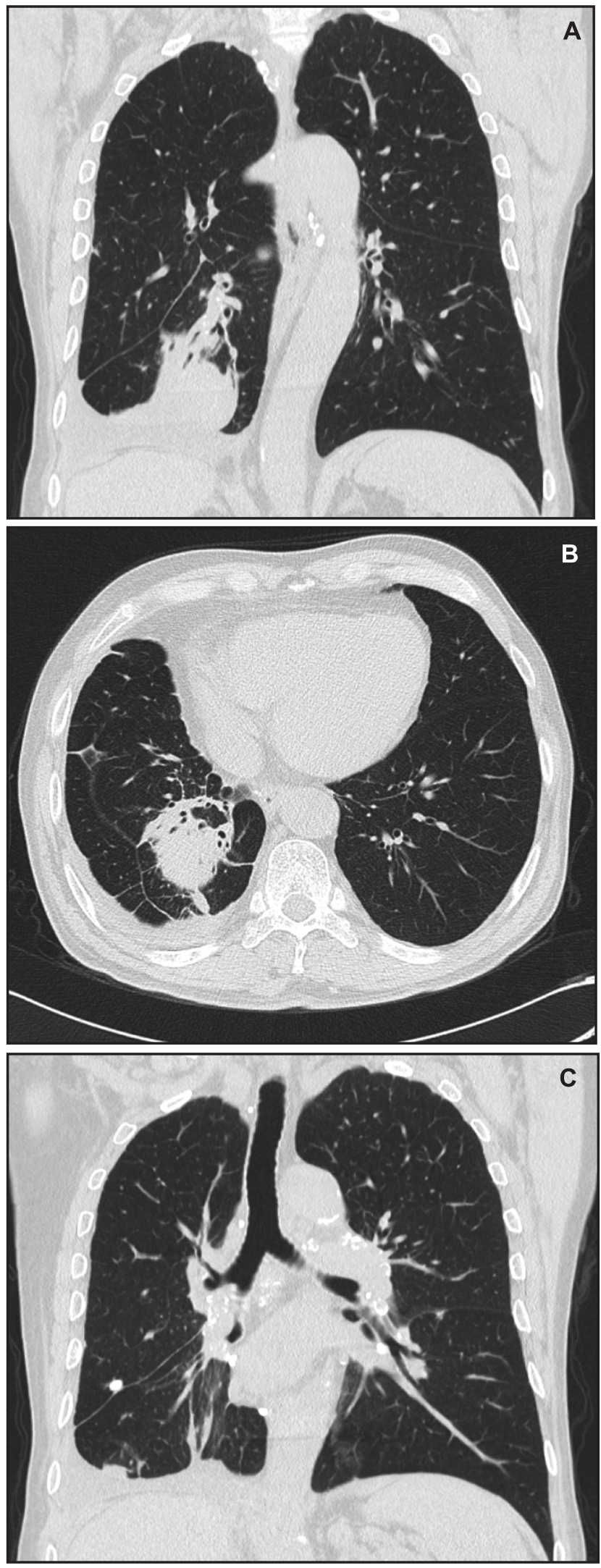

Figura 2.TACAR ventana pulmonar (A) corte coronal y $(B)$ corte axial, que evidencian masa de aproximadamente de $57 * 63 \mathrm{~mm}$ con arterias pulmonares en su interior y alrededor, en contacto con derrame pleural basal importante, compatible con el signo de cola de cometa. (C) corte coronal que evidencia nódulo pulmonar en lóbulo medio de $5.3 \mathrm{~mm}$ de diámetro. 
contaminación sobre una muestra inadecuada, y estudio citológico inespecífico.

Se dio egreso con indicación de controles clínicos e imagenológicos, posterior a lo cual el paciente ha permanecido asintomático.

\section{Discusión}

Las atelectasias redondas constituyen una forma de colapso pulmonar periférico poco común asociado a derrame pleural, reconocido en la literatura (2). Involucra el colapso de parte de un lóbulo no relacionado con la anatomía segmentaria.

Se observó por primera vez en pacientes tratados con colapsoterapia (inducción de neumotórax iatrogénico para el tratamiento de tuberculosis pulmonar cavitada) y entre sus factores causales predominan la historia de exposición a asbestos (29-86\%) y la pleuresía (14-64\%) Tabla 1 (6).

La edad promedio de presentación se sitúa entre $65 \pm$ 13 años, y ocurre principalmente en hombres (>90\%) con historia de tabaquismo (83\%) (7). Constituye una causa rara de nódulo pulmonar solitario en pacientes inmunosuprimidos o no (8). Usualmente cursa sin síntomas o con quejas inespecíficas de tos, disnea, fiebre, pérdida de peso, y raramente dolor pleurítico o hemoptisis. El examen físico con frecuencia es normal o inespecífico, con escasos estertores finos inspiratorios o roce de fricción pleural sobre el área afectada.

Aunque no es bien conocida su patogénesis, tradicionalmente dos teorías han explicado su mecanismo de producción: 1) la teoría del plegamiento (derrame pleural) y 2) la teoría fibrosante (lesión pleural). La primera, establece que el evento inicial es la compresión parenquimatosa y subsecuente invaginación por acumulación locular de líquido pleural, mientras que la segunda propone como evento desencadenante la pleuritis (inflamación local de pleura visceral); cuando se repara, se fibrosa y se contrae. Recientemente surge la teoría de la "distorsión microbronquial"; según ésta, ya sea por presión del líquido pleural y/o contracción de una placa pleural fibrótica, ocurre un desplazamiento del parénquima adyacente y en consecuencia la estrangualción de las vías aéreas pequeñas con absorción de gas distal. Figura 3 (6).

El diagnóstico se establece imagenológicamente; los hallazgos son lo suficientemente característicos, los cuales corresponden generalmente a lesiones únicas, localizadas en lóbulos inferiores, língula o lóbulo medio y el signo de la "cola de cometa", causado como consecuencia del encorvamiento y convergencia de los vasos sanguíneos alrededor de la masa (especificidad 92\%, sensibilidad 83\%), siendo este último el de mayor poder diagnóstico. No obstante, debido a que constituyen masas con realce al contraste, asociadas además con frecuencia a nódulo pulmonar, la diferenciación con neoplasias malignas del pulmón constituyen un reto.

Hasta en la mitad de los casos, la lesión no es detectable, ni adecuadamente caracterizada en radiografía convencional de tórax, lo que hace imperativa la realización de tomografía
Tabla 1. Causas de atelectasia redonda. Tomado de (6)

\begin{tabular}{|l|l|}
\hline Exposición a polvos minerales & $\begin{array}{l}\text { Asbestos (ocupacional y ambiental) } \\
\text { Sílice } \\
\text { Polvos minerales mixtos (ocupacional) }\end{array}$ \\
\hline Derrame pleural exudativo & $\begin{array}{l}\text { Derrame paraneumónico - empiema } \\
\text { Tuberculosis } \\
\text { Hemotórax } \\
\text { Cirugía cardiaca } \\
\text { Hemodiálisis crónica }\end{array}$ \\
\hline Sin involucramiento pleural & $\begin{array}{l}\text { Neumonía por Legionella pneumophila } \\
\text { Histoplasmosis }\end{array}$ \\
& $\begin{array}{l}\text { Insuficiencia renal crónica terminal } \\
\text { Sarcoidosis (?) }\end{array}$ \\
\hline Otras & $\begin{array}{l}\text { Neumotórax (espontáneo, iatrogénico) } \\
\text { Idiopático }\end{array}$ \\
\hline
\end{tabular}

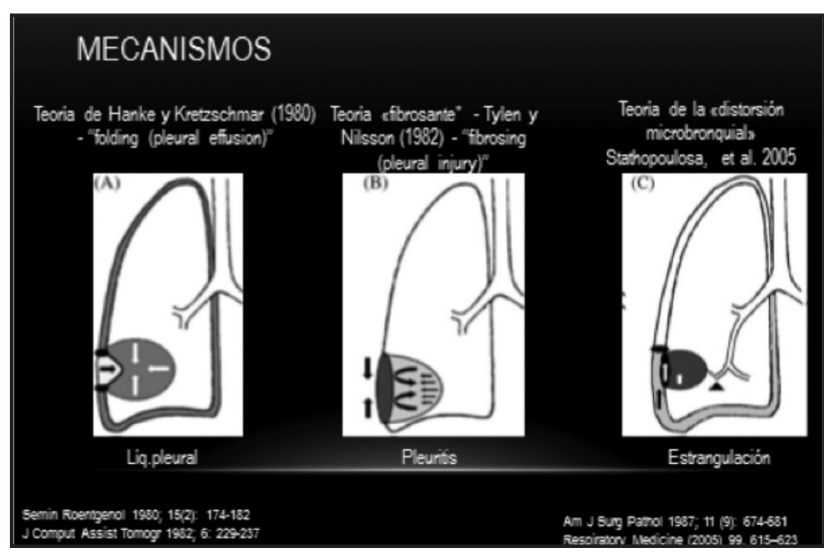

Figura 3. Teorías de la patogénesis de la atelectasia redonda. (Tomado de Georgios T. Stathopoulosa, b, Maria T. Karamessinib, Athanasia E. Sotiriadib, Vasilios G. Pastromasb. Rounded atelectasis of the lung. Respiratory Medicine (2005) 99, 615-623).

computada (TC) en todo paciente con sospecha de atelectasia redonda (Tabla 2). Sólo debe usarse la ecografía cuando no sea factible realizar TC. Por su parte, la resonancia magnética no aporta información adicional comparada con la TC. En los casos dudosos a la TC y cuando su disponibilidad lo permita, puede ser beneficiosa la tomografía por emisión de positrones (PET) para establecer diagnóstico diferencial con

Tabla 2. Signos radiológicos en TC tórax en atelectasia redonda.

\begin{tabular}{|l|l|}
\hline \multicolumn{1}{|c|}{ Signos primarios } & \multicolumn{1}{c|}{ Signos secundarios } \\
\hline $\begin{array}{l}\text { Masa redondeada, en cuña o } \\
\text { lentiforme }\end{array}$ & $\begin{array}{l}\text { Engrosamiento pleural adyacente } \\
\text { Pérdida de volumen pulmonar en lóbulo } \\
\text { aensidad de tejidos blandos, con } \\
\text { broncograma aéreo }\end{array}$ \\
$\begin{array}{l}\text { Tamaño: } 3.5 \text { - 7cm } \\
\text { Localización subpleural }\end{array}$ & $\begin{array}{l}\text { Pesplazamiento de bronquio fuente } \\
\text { Ubicación más común: lóbulo } \\
\text { medio, inferior o língula }\end{array}$ \\
$\begin{array}{l}\text { Marcas broncovasculares con- centro más radiolúcido } \\
\text { vergentes, o signos de la "cola } \\
\text { de cometa" }\end{array}$ & $\begin{array}{l}\text { Aumento de la opacidad del parénquima } \\
\text { adyacente }\end{array}$ \\
Márgenes hiliares difusas/bor- & $\begin{array}{l}\text { Placas pleurales coexistentes, derrame } \\
\text { pleural }\end{array}$ \\
rosas & $\begin{array}{l}\text { Proceso parenquimatoso coexistente (ej. } \\
\text { asbestosis) }\end{array}$ \\
Ángulo agudo entre la masa y la & Nódulos silicóticos dentro de la lesión \\
superficie pleural & Calcificación de la pleura engrosada o de \\
& la propia lesión \\
\hline
\end{tabular}


lesiones malignas; por fortuna, las atelectasias redondas son inactivas metabólicamente y nuevas técnicas de PET con FDG (2-[18F]-fluoro-2-deoxy-D-glucosa alcanzan sensibilidades de $100 \%$ y especificidades de $89 \%$ (6).

Aun cuando las características imagenológicas del paciente presentado fueron típicas, el caso constituyó un reto diagnóstico por factores de "confusión" como su estado de inmunosupresión y la ausencia de historia exposicional a asbestos o cualquier otro polvo mineral. Aunque sus características demográficas concordaron con aquellas típicas reportadas en la literatura, se descartó también como factor causal un proceso infeccioso y al final, no pudo establecerse un factor etiológico, quedando caracterizado como un caso idiopático.

\section{Referencias}

1. Yoshihisa N, Kazuyoshi S, Momoko E, Kumiko A. Rounded Atelectasis that Disappeared after Bronchoscopy. Internal Medicine 1998; (37): 870-871.

2. McHugh K, Blaquiere M. CT Features of Rounded Atelectasis. AJR 1989; 153: 257-260.

3. HH, Loeschke. Storungen des Luftgehalts der Lunge in Henke-Lubaresch. Handbuch der Spezielen pathologischen. Anatomie und Histologie 1928; (3): 559.

4. Hanke R, Kretzschmar R. Round atelectasis. Semin Roentgenol 1980; 15(2): 174-182.

5. M. Martínez Moya, P. Bernáldez Domínguez, M. L. Rodríguez Rodríguez, J Fernández Cruz. El espectro radiologico de las atelectasias redondas: a proposito de 22 observaciones. Neumosur 2000; 11 (4): 21-31.

6. Georgios T. Stathopoulosa, b, Maria T. Karamessinib, Athanasia E. Sotiriadib, Vasilios G. Pastromasb. Rounded atelectasis of the lung. Respiratory Medicine 2005: 99; 615-623.

7. Jara Chinarro B, de Miguel Diez J,Abad Santamaria N, et al. Round atelectasis. Rev Clin Esp 2001; 201(6): 303-7.

8. Martinez-Marcos FJ, Viciana P, Canas E, et al. Etiology of solitary pulmonary nodules in patients with human immunodeficiency virus infection. Clin Infect Dis 1997; 24(5): 908-13 